\title{
SOME PROPERTIES OF ANALYTIC FUNCTIONS ASSOCIATED WITH FRACTIONAL $q$-CALCULUS OPERATORS
}

\author{
H. M. SRIVASTAVA, M. K. AOUF, AND A. O. MOSTAFA
}

Received 24 September, 2019

\begin{abstract}
By applying a fractional $q$-calculus operator, we define the subclasses $\delta_{n}^{\alpha}(\lambda, \beta, b, q)$ and $\mathscr{E}_{n}^{\alpha}(\lambda, \beta, b, q)$ of normalized analytic functions with complex order and negative coefficients. Among the results investigated for each of these function classes, we derive their associated coefficient estimates, radii of close-to-convexity, starlikeness and convexity, extreme points, and growth and distortion theorems.
\end{abstract}

2010 Mathematics Subject Classification: 26A33; 30C45; 33D05

Keywords: analytic functions, fractional $q$-calculus operators, $q$-gamma functions, starlike functions of complex order, convex functions of complex order, close-to-convex functions of complex order, coefficient estimates, radii of close-to-convexity, starlikeness and convexity, extreme points, growth and distortion theorems

\section{INTRODUCTION AND DEFINITIONS}

Here, in this paper, we denote by $\mathcal{A}(n)$ the class of functions of the following normalized form:

$$
f(z)=z+\sum_{k=n+1}^{\infty} a_{k} z^{k} \quad(n \in \mathbb{N} ; \mathbb{N}:=\{1,2,3, \cdots\}),
$$

which are analytic in the open unit disk $\mathbb{U}$ centered at the origin $(z=0)$ in the complex $z$-plane. We write $\mathcal{A}(1)=\mathcal{A}$. We also denote by $\mathcal{T}(n)$ the subclass of $\mathcal{A}(n)$ consisting of functions of the form:

$$
f(z)=z-\sum_{k=n+1}^{\infty} a_{k} z^{k} \quad\left(a_{k} \geqq 0 ; k \geqq n+1 ; n \in \mathbb{N}\right) .
$$

In our investigation, we make use of various operators of $q$-calculus and fractional $q$-calculus. For this purpose, we refer the reader to the various definitions, notations and conventions, which are considerably detailed in our earlier paper (see, for details, [22]; see also [8]). 
For a fixed $\mu \in \mathbb{C}$, a set $\mathbb{D}$ is called a $\mu$-geometric set if and only if both $z \in \mathbb{D}$ and $\mu z \in \mathbb{D}$. For a function $f$ defined on a $q$-geometric set, we make use of Jackson's $q$-derivative and $q$-integral $(0<q<1)$ of a function on a subset of $\mathbb{C}$, which are already introduced in several earlier investigations (see, for example, [2], [4], [6], [8], [9], [10], [14], [15], [16], [17], [21], [22] and [25]).

Now, for a complex-valued function $f(z)$, we introduce the fractional $q$-calculus operators as follows (see, for example, [12] and [13]; see also [1]).

Definition 1 (Fractional $q$-integral operator). The fractional $q$-integral operator $I_{q, z}^{\lambda}$ of order $\lambda$ is defined, for a function $f(z)$, by

$$
I_{q, z}^{\lambda} f(z)=D_{q, z}^{-\lambda} f(z)=\frac{1}{\Gamma_{q}(\lambda)} \int_{0}^{z}(z-t q)_{\lambda-1} f(t) d_{q} t \quad(\lambda>0),
$$

where the function $f(z)$ is analytic in a simply-connected region of the complex $z$-plane containing the origin. Here, and elsewhere in this paper, the $q$-binomial $(z-t q)_{\lambda-1}$ is given by

$$
\begin{array}{r}
(z-t q)_{\lambda-1}= \\
z^{\lambda-1} \prod_{k=0}^{\infty}\left[\frac{1-\left(t q z^{-1}\right) q^{k}}{1-\left(t q z^{-1}\right) q^{\lambda+k-1}}\right] \\
=z^{\lambda}{ }_{1} \Phi_{0}\left(q^{1-\lambda} ;-; q, t q^{\lambda} z^{-1}\right) .
\end{array}
$$

Remark 1 . The $q$-hypergeometric series ${ }_{1} \Phi_{0}(\lambda ;-; q, z)$ is known to be singlevalued when $|\arg (z)|<\pi$ (see, for example, [8]). Therefore, the $q$-binomial $(z-$ $t q)_{\lambda-1}$ in (1.4) is single-valued when

$$
\left|\arg \left(-t q^{\lambda} z^{-1}\right)\right|<\pi, \quad\left|\frac{t q^{\lambda}}{z}\right|<1 \text { and }|\arg (z)|<\pi .
$$

Definition 2 (Fractional $q$-derivative operator). The fractional $q$-derivative operator $D_{q, z}^{\lambda}$ of order $\lambda(0 \leqq \lambda<1)$ is defined, for a function $f(z)$, by

$$
D_{q, z}^{\lambda} f(z)=D_{q, z} I_{q, z}^{1-\lambda} f(z)=\frac{1}{\Gamma_{q}(1-\lambda)} D_{q} \int_{0}^{z}(z-t q)_{-\lambda} f(t) d_{q} t,
$$

where $f(z)$ is suitably constrained and the multiplicity of $(z-t q)_{-\lambda}$ is removed as in Definition 1.

Definition 3 (Extended fractional $q$-derivative operator). Under the hypotheses of Definition 2, for a function $f(z)$, the fractional $q$-derivative of order $\lambda$ is defined by

$$
D_{q, z}^{\lambda} f(z)=D_{q, z}^{m} I_{q, z}^{m-\lambda} f(z) \quad(m-1 \leqq \lambda<1 ; m \in \mathbb{N}) .
$$

Clearly, we have

$$
D_{q, z}^{\lambda} z^{n}=\frac{\Gamma_{q}(n+1)}{\Gamma_{q}(n+1-\lambda)} z^{n-\lambda} \quad(\lambda \geqq 0 ; n>-1) .
$$


Now, by using the operator $D_{q, z}^{\lambda}$, we define (for $-\infty<\lambda<2,0<q<1$ and $z \in \mathbb{U}$, ) a $q$-differintegral operator $\Omega_{q, z}^{\lambda}: \mathcal{T}(n) \rightarrow \mathcal{T}(n)$ as follows (see [12] and [13]):

$$
\Omega_{q, z}^{\lambda} f(z)=\frac{\Gamma_{q}(2-\lambda)}{\Gamma_{q}(\lambda)} z^{\lambda} D_{q, z}^{\lambda} f(z)=z-\sum_{k=n+1}^{\infty} A_{q}(\lambda, k) a_{k} z^{k}
$$

where

$$
A_{q}(\lambda, k)=\frac{\Gamma_{q}(k+1) \Gamma_{q}(2-\lambda)}{\Gamma_{q}(2) \Gamma_{q}(k+1-\lambda)}
$$

and $D_{q, z}^{\lambda} f(z)$ in (1.7) represents, respectively, the fractional $q$-integral of $f(z)$ of order $\lambda(-\infty<\lambda<0)$ and the fractional $q$-derivative of $f(z)$ of order $\lambda(0 \leqq \lambda<2)$ (see, for details, [7,18-20]). We note that some interesting special and limit cases of (1.7) were investigated in the earlier works by Owa and Srivastava [11] and by Srivastava and Owa (see [23] and [24]).

Remark 2. From (1.3), (1.7) and (1.8), we find that

$$
\begin{aligned}
\Omega_{q, z}^{-\lambda} f(z) & =\frac{\Gamma_{q}(2+\lambda)}{\Gamma_{q}(2)} z^{-\lambda} D_{q, z}^{-\lambda} f(z)=\frac{\Gamma_{q}(2+\lambda)}{\Gamma_{q}(2)} z^{-\lambda} I_{q, z}^{\lambda} f(z) \\
& =z-\sum_{k=n+1}^{\infty} A_{q}(-\lambda, k) a_{k} z^{k},
\end{aligned}
$$

where

$$
A_{q}(-\lambda, k)=\frac{\Gamma_{q}(k+1) \Gamma_{q}(2+\lambda)}{\Gamma_{q}(2) \Gamma_{q}(k+1+\lambda)} \quad(\lambda>0 ; 0<q<1) .
$$

Definition 4. A function $f(z) \in \mathcal{T}(n)$ is said to be in the function class:

$$
\delta_{n}^{\alpha}(\lambda, \beta, b, q) \quad\left(\lambda<2 ; 0 \leqq \alpha \leqq 1 ; 0<q<1 ; \beta>0 ; b \in \mathbb{C}^{*}=\mathbb{C} \backslash\{0\}\right)
$$

if it satisfies the following condition:

$$
\left|\frac{1}{b}\left(\frac{(1-\alpha) z D_{q}\left(\Omega_{q, z}^{\lambda} f(z)\right)+\alpha z D_{q}\left(z D_{q}\left(\Omega_{q, z}^{\lambda} f(z)\right)\right)}{(1-\alpha) \Omega_{q, z}^{\lambda} f(z)+\alpha z D_{q}\left(\Omega_{q, z}^{\lambda} f(z)\right)}-1\right)\right|<\beta .
$$

Some of the interesting particular cases of the function class $\rho_{n}^{\alpha}(\lambda, \beta, b, q)$ are being recorded below:

(i) $\delta_{n}^{\alpha}(\lambda, 1, b, q)=\wp_{n}^{\alpha}(\lambda, b, q) \quad$ (see [12]);

(ii) $\delta_{n}^{\alpha}(0, \beta, b, q)=\wp_{n}^{\alpha}(\beta, b, q)$, where

$$
8_{n}^{\alpha}(\beta, b, q):=\{f: f \in T(n) \quad \text { and }
$$




$$
\left.\left|\frac{1}{b}\left(\frac{(1-\alpha) z D_{q} f(z)+\alpha z^{2} D_{q}^{2} f(z)}{(1-\alpha) f(z)+\alpha z D_{q} f(z)}-1\right)\right|<\beta\right\} .
$$

(iii) $\lim _{q \rightarrow 1-} f_{n}^{\alpha}(\beta, b, q)=\wp_{n}(b, \alpha, \beta)$ (see [3]);

(iv) $\delta_{n}^{0}(\lambda, \beta, b, q)=\wp_{n}^{*}(\lambda, \beta, b, q)$, where

$$
\S_{n}(b, \alpha, \beta):=\left\{f: f \in \mathcal{T}(n) \text { and }\left|\frac{1}{b}\left(\frac{z D_{q}\left(\Omega_{q, z}^{\lambda} f(z)\right)}{\Omega_{q, z}^{\lambda} f(z)}-1\right)\right|<\beta\right\} ;
$$

(v) $\lim _{q \rightarrow 1-} 8_{n}^{*}(\lambda, \beta, b, q)=\mathcal{K}_{n}(\lambda, b, \beta)$ (see [5] with $\left.p=1\right)$;

(vi) $\delta_{n}^{1}(\lambda, \beta, b, q)=\ell_{n}(\lambda, \beta, b, q)$, where

$$
\digamma_{n}(\lambda, \beta, b, q):=\left\{f: f \in \mathcal{T}(n) \quad \text { and } \quad\left|\frac{1}{b}\left(\frac{z D_{q}^{2}\left(\Omega_{q, z}^{\lambda} f(z)\right)}{D_{q}\left(\Omega_{q, z}^{\lambda} f(z)\right)}-1\right)\right|<\beta\right\} \text {. }
$$

Definition 5. A function $f(z) \in \mathcal{T}(n)$ is in the function class

$$
\mathcal{E}_{n}^{\alpha}(\lambda, \beta, b, q)\left(\lambda<2 ; 0 \leqq \alpha \leqq 1 ; 0<q<1 ; b \in \mathbb{C}^{*} ; \beta>0\right)
$$

if it satisfies the following condition:

$$
\left|\frac{1}{b}\left(D_{q}\left(\Omega_{q, z}^{\lambda} f(z)\right)+\alpha z D_{q}^{2}\left(\Omega_{q, z}^{\lambda} f(z)\right)-1\right)\right|<\beta .
$$

We choose to note the following special case of the function class $\mathcal{E}_{n}^{\alpha}(\lambda, \beta, b, q)$ :

(i) $\mathcal{G}_{n}^{\alpha}(0, \beta, b, q)=\mathscr{\mathcal { E }}_{n}^{\alpha}(\beta, b, q)$, where

$$
\mathcal{E}_{n}^{\alpha}(\beta, b, q)=\left\{f: f \in \mathcal{T}(n) \quad \text { and } \quad\left|\frac{1}{b}\left(D_{q} f(z)+\alpha z D_{q}^{2} f(z)-1\right)\right|<\beta\right\} ;
$$

(ii) $\mathcal{E}_{n}^{\alpha}(\lambda, 1, b, q)=\mathcal{R}_{n}^{\alpha}(\lambda, b, q)$ (see [13]);

(iii) $\mathcal{G}_{n}^{\alpha}(0, \beta, b, q)=\mathcal{R}_{n}(\alpha, \beta, b, q) \quad$ (see [13]);

(iv) $\lim _{q \rightarrow 1-} \mathcal{E}_{n}^{\alpha}(0, \beta, b, q)=\mathcal{R}_{n}(\alpha, \beta, b)$ (see [3]).

For each of the above-defined general function classes $\delta_{n}^{\alpha}(\lambda, \beta, b, q)$ and $\mathcal{E}_{n}^{\alpha}(\lambda, \beta, b, q)$ of analytic functions with complex order and negative coefficients, we propose here to investigate the associated coefficient estimates, radii of closeto-convexity, starlikeness and convexity, extreme points, and growth and distortion theorems. 
2. Properties of the Function Classes $\mathcal{P}_{n}^{\alpha}(\lambda, \beta, b, q)$ AND $\mathscr{E}_{n}^{\alpha}(\lambda, \beta, b, q)$

Henceforth in this paper, unless otherwise mentioned, we assume that $\lambda<2,0 \leqq$ $\alpha \leqq 1,0<q<1, b \in \mathbb{C}^{*}, \beta>0,[\lambda]_{q}$ denotes the basic (or $q$-) number defined by

$$
[\lambda]_{q}=\frac{1-q^{\lambda}}{1-q} \quad(|q|<1),
$$

which readily yields

$$
[\lambda]_{q}=\frac{1-q^{\lambda}}{1-q} \rightarrow \lambda \quad(q \rightarrow 1-),
$$

$A_{q}(\lambda, k)$ is given by (1.8), $f(z)$ is in the form (1.2) and $z \in \mathbb{U}$.

Theorem 1. The function $f(z) \in \wp_{n}^{\alpha}(\lambda, \beta, b, q)$ if and only if

$$
\sum_{k=n+1}^{\infty}\left([k]_{q}+\beta|b|-1\right)\left[1+\alpha\left([k]_{q}-1\right)\right] A_{q}(\lambda, k) a_{k} \leqq \beta|b| .
$$

Proof. Let $f(z) \in \mathcal{S}_{n}^{\alpha}(\lambda, \beta, b, q)$. Then, in view of (1.11) and (1.7), we readily find that

$$
\Re\left(\frac{-\sum_{k=n+1}^{\infty}\left[1+\alpha\left([k]_{q}-1\right)\right]\left([k]_{q}-1\right) A_{q}(\lambda, k) a_{k} z^{k-1}}{1-\sum_{k=n+1}^{\infty}\left[1+\alpha\left([k]_{q}-1\right)\right] A_{q}(\lambda, k) a_{k} z^{k-1}}\right)>-\beta|b| .
$$

Setting $z=r(0 \leqq r<1)$ in (2.3), we observe that the expression in the denominator of the left-hand side of (2.3) is positive for $r=0$ and also for $0<r<1$. Thus, if we let $r \rightarrow 1$ - through real values, (2.3) would lead us to (2.2).

Conversely, let (2.2) hold true and $|z|=1$. We then find that

$$
\begin{gathered}
\left|\frac{(1-\alpha) z D_{q}\left(\Omega_{q, z}^{\lambda} f(z)\right)+\alpha z D_{q}\left(z D_{q}\left(\Omega_{q, z}^{\lambda} f(z)\right)\right)}{(1-\alpha) \Omega_{q, z}^{\lambda} f(z)+\alpha z D_{q}\left(\Omega_{q, z}^{\lambda} f(z)\right)}-1\right| \\
\leqq \frac{\beta|b|\left\{1-\sum_{k=n+1}^{\infty}\left[1+\alpha\left([k]_{q}-1\right)\right] A_{q}(\lambda, k) a_{k}\right\}}{1-\sum_{k=n+1}^{\infty}\left[1+\alpha\left([k]_{q}-1\right)\right] A_{q}(\lambda, k) a_{k}}=\beta|b| .
\end{gathered}
$$

Hence, by the Maximum Modulus Theorem, we conclude that $f(z) \in \wp_{n}^{\alpha}(\lambda, \beta, b, q)$, which completes the proof of Theorem 1.

The following corollary follows easily from Theorem 1. 
Corollary 1. Let $f(z) \in \mathcal{S}_{n}^{\alpha}(\lambda, \beta, b, q)$. Then

$$
a_{k} \leqq \frac{\beta|b|}{\left([k]_{q}+\beta|b|-1\right)\left[1+\alpha\left([k]_{q}-1\right)\right] A_{q}(\lambda, k)} \quad(k \geqq n+1) .
$$

The result is sharp for the function $f(z)$ given (for $(k \geqq n+1)$ by

$$
f(z)=z-\frac{\beta|b|}{\left([k]_{q}+\beta|b|-1\right)\left[1+\alpha\left([k]_{q}-1\right)\right] A_{q}(\lambda, k)} z^{k} .
$$

Putting $\beta=1$ in Theorem 1, we have Corollary 2 below.

Corollary 2. Let $f(z) \in \oiint_{n}^{\alpha}(\lambda, b, q)$. Then

$$
\sum_{k=n+1}^{\infty}\left([k]_{q}+|b|-1\right)\left[1+\alpha\left([k]_{q}-1\right)\right] A_{q}(\lambda, k) a_{k} \leqq|b| .
$$

Corollary 3. Let $f(z) \in \mathcal{S}_{n}^{\alpha}(\lambda, b, q)$. Then

$$
a_{k} \leqq \frac{|b|}{\left([k]_{q}+|b|-1\right)\left[1+\alpha\left([k]_{q}-1\right)\right] A_{q}(\lambda, k)} \quad(k \geqq n+1) .
$$

The result is sharp for the function $f(z)$ given by

$$
f(z)=z-\frac{|b|}{\left([k]_{q}+|b|-1\right)\left[1+\alpha\left([k]_{q}-1\right)\right] A_{q}(\lambda, k)} z^{k} \quad(k \geqq n+1) .
$$

It is not difficult to prove the following results. The details involved are being left as an exercise for the interested reader.

Theorem 2. The function $f(z) \in \mathscr{G}_{n}^{\alpha}(\lambda, \beta, b, q)$ if and only if

$$
\sum_{k=n+1}^{\infty}[k]_{q}\left[1+\alpha\left([k]_{q}-1\right)\right] A_{q}(\lambda, k) a_{k} \leqq \beta|b| .
$$

Corollary 4. Let $f(z) \in \mathcal{E}_{n}^{\alpha}(\lambda, \beta, b, q)$. Then

$$
a_{k} \leqq \frac{\beta|b|}{[k]_{q}\left[1+\alpha\left([k]_{q}-1\right)\right] A_{q}(\lambda, k)} .
$$

The result is sharp for the function $f(z)$ given by

$$
f(z)=z-\frac{\beta|b|}{[k]_{q}\left[1+\alpha[k]_{q}-1\right] A_{q}(\lambda, k)} z^{k} \quad(k \geqq n+1) .
$$

We now state (without proof) Theorem 3 below.

Theorem 3. If $b_{1}, b_{2} \in \mathbb{C}^{*}$ and $\left|b_{1}\right|<\left|b_{2}\right|$, then

$$
\wp_{n}^{\alpha}\left(\lambda, \beta, b_{1}, q\right) \subset \wp_{n}^{\alpha}\left(\lambda, \beta, b_{2}, q\right) .
$$

The following result can indeed be proven along the lines which we have already indicated above. 
Theorem 4. If $b_{1}, b_{2} \in \mathbb{C}^{*}$ and $\left|b_{1}\right|<\left|b_{2}\right|$, then

$$
\mathscr{E}_{n}^{\alpha}\left(\lambda, \beta, b_{1}, q\right) \subset \mathscr{E}_{n}^{\alpha}\left(\lambda, \beta, b_{2}, q\right) .
$$

3. EXTREME POINTS FOR THE FUNCTION CLASSES $\wp_{n}^{\alpha}(\lambda, \beta, b, q)$ AND

$$
\mathscr{E}_{n}^{\alpha}(\lambda, \beta, b, q)
$$

In this section, we first prove the following result.

Theorem 5. Let $f_{n}(z)=z$ and

$$
\begin{gathered}
f_{k}(z)=z-\frac{\beta|b|}{\left([k]_{q}+\beta|b|-1\right)\left[1+\alpha\left([k]_{q}-1\right)\right] A_{q}(\lambda, k)} z^{k} \\
(k \geqq n+1) .
\end{gathered}
$$

Then the function $f(z)$ is in the class $\wp_{n}^{\alpha}(\lambda, \beta, b, q)$ if and only if it can be expressed in the following form:

$$
f(z)=\sum_{k=n}^{\infty} \mu_{k} f_{k}(z)
$$

where

$$
\sum_{k=n}^{\infty} \mu_{k}=1 \quad \text { and } \quad \mu_{k} \geqq 0 .
$$

Proof. By assuming (3.2) to hold true, if we appropriately apply Theorem 1, it is not difficult to conclude that $f(z) \in \delta_{n}^{\alpha}(\lambda, \beta, b, q)$.

Conversely, upon leting $f(z) \in \mathcal{S}_{n}^{\alpha}(\lambda, \beta, b, q)$, if we set

$$
\mu_{k}=\frac{\left([k]_{q}+\beta|b|-1\right)\left[1+\alpha\left([k]_{q}-1\right)\right] A_{q}(\lambda, k)}{\beta|b|} a_{k} \quad(k \geqq n+1)
$$

and

$$
\mu_{n}=1-\sum_{k=n+1}^{\infty} \mu_{k},
$$

we can easily see that $f(z)$ can be expressed in the form (3.2). This completes the proof of Theorem 5 .

Corollary 5. The extreme points of the function class $\gamma_{n}^{\alpha}(\lambda, \beta, b, q)$ are the functions $f_{n}(z)=z$ and $f_{k}(z)(k \geqq n+1)$ given by $(3.1)$.

Similarly, we can prove the following theorem.

Theorem 6. Let $f_{n}(z)=z$ and

$$
f_{k}(z)=z-\frac{\beta|b|}{[k]_{q}\left[1+\alpha\left([k]_{q}-1\right)\right] A_{q}(\lambda, k)} z^{k} \quad(k \geqq n+1) .
$$


Then the function $f(z)$ is in the class $\beta_{n}^{\alpha}(\lambda, \beta, b, q)$ if and only if it can be expressed in the form given by

$$
f(z)=\sum_{k=n}^{\infty} \mu_{k} f_{k}(z)
$$

where

$$
\sum_{k=n}^{\infty} \mu_{k}=1 \quad \text { and } \quad \mu_{k} \geqq 0 .
$$

Corollary 6. The extreme points of the function class $\mathscr{E}_{n}^{\alpha}(\lambda, \beta, b, q)$ are the functions $f_{n}(z)=z$ and $f_{k}(z)(k \geqq n+1)$ given by (3.3).

\section{RADII OF CLOSE-TO-CONVEXITY, STARLIKENESS AND CONVEXITY OF THE}

$$
\text { FUNCTION CLASS } \gamma_{n}^{\alpha}(\lambda, \beta, b, q)
$$

Theorem 7. Let $f(z) \in \wp_{n}^{\alpha}(\lambda, \beta, b, q)$. Then $f(z)$ is close-to-convex of order $\rho(0 \leqq \rho<1)$ in $|z|<r_{1}$, where

$$
r_{1}:=\inf _{k \geqq n+1}\left\{\frac{(1-\rho)\left([k]_{q}+\beta|b|-1\right)\left[1+\alpha\left([k]_{q}-1\right)\right] A_{q}(\lambda, k)}{k \beta|b|}\right\}^{\frac{1}{k-1}} .
$$

The sharpness of this result is attained for the function $f(z)$ given by (2.5).

Proof. By showing that

$$
\left|f^{\prime}(z)-1\right| \leqq 1-\rho \quad \text { for } \quad|z|<r_{1},
$$

where $r_{1}$ is given by (4.1), we readily find that

$$
\left|f^{\prime}(z)-1\right| \leqq 1-\rho,
$$

if

$$
\sum_{k=n+1}^{\infty} \frac{k}{1-\rho} a_{k}|z|^{k-1} \leqq 1 .
$$

But, by Theorem 1, it is seen that (4.2) will hold true if (for $k \geqq n+1$ )

$$
|z| \leqq\left(\frac{(1-\rho)\left([k]_{q}+\beta|b|-1\right)\left[1+\alpha\left([k]_{q}-1\right)\right] A_{q}(\lambda, k)}{k \beta|b|}\right)^{\frac{1}{k-1}} .
$$

This completes the proof of Theorem 7 .

By using arguments and analysis similar to those in the proof of Theorem 7, we can analogously derive Theorem 8 and Corollary 7 below. 
Theorem 8. Let $f(z) \in \vartheta_{n}^{\alpha}(\lambda, \beta, b, q)$. Then the function $f(z)$ is starlike of order $\rho(0 \leqq \rho<1)$ in $|z|<r_{2}$, where

$$
r_{2}:=\inf _{k \geqq n+1}\left\{\frac{(1-\rho)\left([k]_{q}+\beta|b|-1\right)\left[1+\alpha\left([k]_{q}-1\right)\right] A_{q}(\lambda, k)}{(k-\rho) \beta|b|}\right\}^{\frac{1}{k-1}} .
$$

The sharpness of this result is attained for the function $f(z)$ given by (2.5).

Corollary 7. Let $f(z) \in 8_{n}^{\alpha}(\lambda, \beta, b, q)$. Then the function $f(z)$ is convex of order $\rho(0 \leqq \rho<1)$ in $|z|<r_{3}$, where

$$
r_{3}:=\inf _{k \geqq n+1}\left\{\frac{(1-\rho)\left([k]_{q}+\beta|b|-1\right)\left[1+\alpha\left([k]_{q}-1\right)\right] A_{q}(\lambda, k)}{k(k-\rho) \beta|b|}\right\}^{\frac{1}{k-1}} .
$$

The sharpness of the result is attained for the function $f(z)$ given by (2.5).

\section{GROWTH AND DISTORTION THEOREMS}

For convenience in this section, for $k \geqq n+1$, we shall henceforth use the following notations:

$$
\sigma_{k, \alpha}(\lambda, \beta, b, q):=\left([k]_{q}+\beta|b|-1\right)\left[1+\alpha\left([k]_{q}-1\right)\right] A_{q}(\lambda, k)
$$

and

$$
\phi_{k, \alpha}(\lambda, \beta, b, q):=[k]_{q}\left[1+\alpha\left([k]_{q}-1\right)\right] A_{q}(\lambda, k) .
$$

We now prove the following which will be needed in our further investigation in this section.

Lemma 1. The sequence $\left\{A_{q}(\lambda, k)\right\}_{k=n+1}^{\infty}$ is a decreasing sequence in $k(k \geqq n+1)$ for $\lambda<2$ and $0<q<1$.

Proof. It follows from (1.8) and the recurrence relation:

$$
\Gamma_{q}(z+1)=[z]_{q} \Gamma_{q}(z)
$$

that

$$
\begin{aligned}
\frac{A_{q}(\lambda, k+1)}{A_{q}(\lambda, k)} & =\frac{\Gamma_{q}(k+2) \Gamma_{q}(k-\lambda+1)}{\Gamma_{q}(k+1) \Gamma_{q}(k-\lambda+2)} \\
& =\frac{[k+1]_{q} \Gamma_{q}(k+1) \Gamma_{q}(k-\lambda+1)}{\Gamma_{q}(k+1)[k-\lambda+1]_{q} \Gamma_{q}(k-\lambda+2)}=\frac{[k+1]_{q}}{[k-\lambda+1]_{q}} .
\end{aligned}
$$

It is sufficient to consider the value $k=n+1$. By using the definition (2.1) of the basic (or $q$-) number $[\lambda]_{q}$ again, we thus find that

$$
\frac{A_{q}(\lambda, k+1)}{A_{q}(\lambda, k)}=\frac{[n+2]_{q}}{[n-\lambda+2]_{q}}=\frac{1-q^{n+2}}{1-q^{n-\lambda+2}} \quad(0<q<1 ;-\infty<\lambda<2) .
$$


The sequence $\left\{A_{q}(\lambda, k)\right\}_{k=n+1}^{\infty}$ is a decreasing sequence in $k$ if

$$
\frac{A_{q}(\lambda, k+1)}{A_{q}(\lambda, k)}<1 \quad(k \geqq n+1),
$$

that is, if

$$
\frac{1-q^{n+2}}{1-q^{n-\lambda+2}}<1 \quad(0<q<1 ;-\infty<\lambda<2),
$$

which implies that $\lambda<0$. Thus $\left\{A_{q}(\lambda, k)\right\}_{k=n+1}^{\infty}$ is a decreasing sequence in $k$ ( $k \geqq$ $n+1)$ for $-\infty<\lambda<2$ and $0<q<1$.

Theorem 9. Let $f(z) \in \wp_{n}^{\alpha}(\lambda, \beta, b, q)$. Then

$$
|z|-\frac{\beta|b|}{\sigma_{n+1, \alpha}(\lambda, \beta, b, q)}|z|^{n+1} \leqq|f(z)| \leqq|z|+\frac{\beta|b|}{\sigma_{n+1, \alpha}(\lambda, \beta, b, q)}|z|^{n+1} .
$$

The result is sharp for the function $f(z)$ given by

$$
f(z)=z-\frac{\beta|b|}{\sigma_{n+1, \alpha}(\lambda, \beta, b, q)} z^{n+1} .
$$

Proof. Since $f(z) \in \wp_{n}^{\alpha}(\lambda, \beta, b, q)$, in view of Theorem 1, we have

$$
\sigma_{n+1, \alpha}(\lambda, \beta, b, q) \sum_{k=n+1}^{\infty} a_{k} \leqq \sum_{k=n+1}^{\infty} \sigma_{k, \alpha}(\lambda, \beta, b, q) a_{k} \leqq \beta|b|,
$$

that is,

$$
\sum_{k=n+1}^{\infty} a_{k} \leqq \frac{\beta|b|}{\sigma_{n+1, \alpha}(\lambda, \beta, b, q)}
$$

We thus obtain

$$
\begin{aligned}
|f(z)| & \geqq|z|-\sum_{k=n+1}^{\infty} a_{k}|z|^{k} \geqq|z|-|z|^{n+1} \sum_{k=n+1}^{\infty} a_{k} \\
& \geqq|z|-\frac{\beta|b|}{\sigma_{n+1, \alpha}(\lambda, \beta, b, q)}|z|^{n+1}
\end{aligned}
$$

and

$$
\begin{aligned}
|f(z)| & \leqq|z|+\sum_{k=n+1}^{\infty} a_{k}|z|^{k} \leqq|z|+|z|^{n+1} \sum_{k=n+1}^{\infty} a_{k} \\
& \leqq|z|+\frac{\beta|b|}{\sigma_{n+1, \alpha}(\lambda, \beta, b, q)}|z|^{n+1} .
\end{aligned}
$$

These last inequalities (5.7) and (5.8) complete the proof of Theorem 9. 
Corollary 8. Under the hypothesis of Theorem 9 , the function $f(z)$ is included in a disk with center at the origin and radius $r$ given by

$$
r=1+\frac{\beta|b|}{\sigma_{n+1, \alpha}(\lambda, \beta, b, q)} .
$$

Similarly, we can prove the following distortion theorem for $f(z) \in \mathcal{E}_{n}^{\alpha}(\lambda, \beta, b, q)$.

Theorem 10. Let $f(z) \in \mathcal{G}_{n}^{\alpha}(\lambda, \beta, b, q)$ and let $\phi_{k, \alpha}(\lambda, \beta, b, q)$ be given by (5.2). Then

$$
|z|-\frac{\beta|b|}{\phi_{n+1, \alpha}(\lambda, \beta, b, q)}|z|^{n+1} \leqq|f(z)| \leqq|z|+\frac{\beta|b|}{\phi_{n+1, \alpha}(\lambda, \beta, b, q)}|z|^{n+1} .
$$

The result is sharp for the function $f(z)$ given by

$$
f(z)=z-\frac{\beta|b|}{\phi_{n+1, \alpha}(\lambda, \beta, b, q)} z^{n+1} .
$$

Corollary 9. Under the hypothesis of Theorem 10, the function $f(z)$ is included in a disk with its center at the origin and its radius $r$ given by

$$
r=1+\frac{\beta|b|}{\phi_{n+1, \alpha}(\lambda, \beta, b, q)} .
$$

A further distortion theorem involving the generalized fractional $q$-differintegral operator $\Omega_{q, z}^{\lambda}$ defined by (1.7) is given by the following theorem.

Theorem 11. Let $f(z) \in \wp_{n}^{\alpha}(\lambda, \beta, b, q)$. Then

$$
\begin{aligned}
|z| & -\frac{\beta|b|}{\left([n+1]_{q}+\beta|b|-1\right)\left[1+\alpha\left([n+1]_{q}-1\right)\right]}|z|^{n+1} \\
& \leqq\left|\Omega_{q, z}^{\lambda} f(z)\right| \\
& \leqq|z|+\frac{\beta|b|}{\left([n+1]_{q}+\beta|b|-1\right)\left[1+\alpha\left([n+1]_{q}-1\right)\right]}|z|^{n+1} .
\end{aligned}
$$

The result is sharp.

Proof. From the above Lemma 1, in conjunction with the equations (5.6) and (1.7), we have

$$
\begin{aligned}
\left|\Omega_{q, z}^{\lambda} f(z)\right| & \geqq|z|-A_{q}(\lambda, n+1)|z|^{n+1} \sum_{k=n+1}^{\infty} a_{k} \\
& \geqq|z|-\frac{\beta|b|}{\left([n+1]_{q}+\beta|b|-1\right)\left[1+\alpha\left([n+1]_{q}-1\right)\right]}|z|^{n+1}
\end{aligned}
$$

and

$$
\left|\Omega_{q, z}^{\lambda} f(z)\right| \leqq|z|+A_{q}(\lambda, n+1)|z|^{n+1} \sum_{k=n+1}^{\infty} a_{k}
$$




$$
\leqq|z|+\frac{\beta|b|}{\left([n+1]_{q}+\beta|b|-1\right)\left[1+\alpha\left([n+1]_{q}-1\right)\right]}|z|^{n+1} .
$$

The equalities in (5.11) are attained for the function $f(z)$ given by

$$
\begin{aligned}
D_{q, z}^{\lambda} f(z) & =\frac{\Gamma_{q}(z) z^{1-\lambda}}{\Gamma_{q}(2-\lambda)} \\
& \cdot\left(1-\frac{\beta|b|}{\left([n+1]_{q}+\beta|b|-1\right)\left[1+\alpha\left([n+1]_{q}-1\right)\right]}|z|^{n}\right)
\end{aligned}
$$

or by the function $f(z)$ given by (5.5). We have thus completed our demonstration of Theorem 11.

From Theorem 10 and (1.7), we have the following distortion inequality involving the fractional $q$-derivative operator $D_{q, z}^{\lambda}$.

Corollary 10. Let $f(z) \in \wp_{n}^{\alpha}(\lambda, \beta, b, q)$. Then

$$
\begin{gathered}
\frac{\Gamma_{q}(2)}{\Gamma_{q}(2-\lambda)}|z|^{1-\lambda}\left(1-\frac{\beta|b|}{\left([n+1]_{q}+\beta|b|-1\right)\left[1+\alpha\left([n+1]_{q}-1\right)\right]}|z|^{n}\right) \\
\leqq\left|D_{q, z}^{\lambda} f(z)\right| \leqq \frac{\Gamma_{q}(2)}{\Gamma_{q}(2-\lambda)}|z|^{1-\lambda} \\
\cdot\left(1+\frac{\beta|b|}{\left([n+1]_{q}+\beta|b|-1\right)\left[1+\alpha\left([n+1]_{q}-1\right)\right]}|z|^{n}\right) .
\end{gathered}
$$

The result is sharp for the function $f(z)$ given by (5.5).

Upon setting $\beta=1$ in Corollary 10 , we get the following corollary which provided the corrected version of a result obtained by Purohit and Raina [12, Corollary 1].

Corollary 11. Let $f(z) \in \wp_{n}^{\alpha}(\lambda, b, q)$. Then

$$
\begin{gathered}
\frac{\Gamma_{q}(2)}{\Gamma_{q}(2-\lambda)}|z|^{1-\lambda}\left(1-\frac{|b|}{\left([n+1]_{q}+|b|-1\right)\left[1+\alpha\left([n+1]_{q}-1\right)\right]}|z|^{n}\right) \\
\leqq\left|D_{q, z}^{\lambda} f(z)\right| \leqq \frac{\Gamma_{q}(2)}{\Gamma_{q}(2-\lambda)}|z|^{1-\lambda} \\
\cdot\left(1+\frac{|b|}{\left([n+1]_{q}+|b|-1\right)\left[1+\alpha\left([n+1]_{q}-1\right)\right]}|z|^{n}\right) .
\end{gathered}
$$

The result is sharp for the function $f(z)$ given by (5.5) with $\beta=1$.

Also, in view of (1.9) or by virtue of (1.3), Theorem 10 gives the following distortion inequality involving the fractional $q$-integral operator $I_{q, z}^{\lambda}$.

Corollary 12. Let $f(z) \in \wp_{n}^{\alpha}(\lambda, \beta, b, q)$. Then

$$
\frac{\Gamma_{q}(2)}{\Gamma_{q}(2+\lambda)}|z|^{1+\lambda}\left(1-\frac{\beta|b|}{\left([n+1]_{q}+\beta|b|-1\right)\left[1+\alpha\left([n+1]_{q}-1\right)\right]}|z|^{n}\right)
$$




$$
\begin{aligned}
\leqq & \left|I_{q, z}^{\lambda} f(z)\right| \frac{\Gamma_{q}(2)}{\Gamma_{q}(2+\lambda)}|z|^{1+\lambda} \\
& \cdot\left(1+\frac{\beta|b|}{\left([n+1]_{q}+\beta|b|-1\right)\left[1+\alpha\left([n+1]_{q}-1\right)\right]}|z|^{n}\right) .
\end{aligned}
$$

The result is sharp for the function $f(z)$ given by (5.5).

Putting $\beta=1$ in Corollary 12, we have the following result.

Corollary 13. Let $f(z) \in \wp_{n}^{\alpha}(\lambda, b, q)$. Then

$$
\begin{gathered}
\frac{\Gamma_{q}(2)}{\Gamma_{q}(2+\lambda)}|z|^{1+\lambda}\left(1-\frac{|b|}{\left([n+1]_{q}+|b|-1\right)\left[1+\alpha\left([n+1]_{q}-1\right)\right]}|z|^{n}\right) \\
\leqq\left|I_{q, z}^{\lambda} f(z)\right| \leqq \frac{\Gamma_{q}(2)}{\Gamma_{q}(2+\lambda)}|z|^{1+\lambda} \\
\cdot\left(1+\frac{|b|}{\left([n+1]_{q}+|b|-1\right)\left[1+\alpha\left([n+1]_{q}-1\right)\right]}|z|^{n}\right) .
\end{gathered}
$$

The result is sharp for the function $f(z)$ given by (5.5) with $\beta=1$ and $\lambda$ replaced by $-\lambda$.

Theorem 12. Let $f(z) \in \mathcal{E}_{n}^{\alpha}(\lambda, \beta, b, q)$. Then

$$
\begin{aligned}
|z| & -\frac{\beta|b|}{[n+1]_{q}\left[1+\alpha\left([n+1]_{q}-1\right)\right]}|z|^{n+1} \\
& \leqq\left|\Omega_{q, z}^{\lambda} f(z)\right| \\
& \leqq|z|+\frac{\beta|b|}{[n+1]_{q}\left[1+\alpha\left([n+1]_{q}-1\right)\right]}|z|^{n+1} .
\end{aligned}
$$

The result is sharp for the function $f(z)$ given by

$$
D_{q, z}^{\lambda} f(z)=\frac{\Gamma_{q}(z) z^{1-\lambda}}{\Gamma_{q}(2-\lambda)}\left(1-\frac{\beta|b|}{[n+1]_{q}\left[1+\alpha\left([n+1]_{q}-1\right)\right]}|z|^{n}\right)
$$

or by the function $f(z)$ given by (5.10).

Similarly, we can prove the following distortion inequalities for $f(z) \in \mathcal{E}_{n}^{\alpha}(\lambda, \beta, b, q)$ involving the fractional $q$-derivative operator $D_{q, z}^{\lambda}$ and the fractional $q$-integral operator $I_{q, z}^{\lambda}$.

Corollary 14. Let $f(z) \in G_{n}^{\alpha}(\lambda, \beta, b, q)$. Then

$$
\begin{aligned}
& \frac{\Gamma_{q}(2)}{\Gamma_{q}(2-\lambda)}|z|^{1-\lambda}\left(1-\frac{\beta|b|}{[n+1]_{q}\left[1+\alpha\left([n+1]_{q}-1\right)\right]}|z|^{n}\right) \\
& \quad \leqq\left|D_{q, z}^{\lambda} f(z)\right|
\end{aligned}
$$




$$
\leqq \frac{\Gamma_{q}(2)}{\Gamma_{q}(2-\lambda)}|z|^{1-\lambda}\left(1+\frac{\beta|b|}{[n+1]_{q}\left[1+\alpha\left([n+1]_{q}-1\right)\right]}|z|^{n}\right) .
$$

The result is sharp for the function $f(z)$ given by (5.10).

Corollary 15. Let $f(z) \in \mathcal{E}_{n}^{\alpha}(\lambda, \beta, b, q)$. Then

$$
\begin{aligned}
& \frac{\Gamma_{q}(2)}{\Gamma_{q}(2+\lambda)}|z|^{1+\lambda}\left(1-\frac{\beta|b|}{[n+1]_{q}\left[1+\alpha\left([n+1]_{q}-1\right)\right]}|z|^{n}\right) \\
& \quad \leqq\left|I_{q, z}^{\lambda} f(z)\right| \\
& \quad \leqq \frac{\Gamma_{q}(2)}{\Gamma_{q}(2+\lambda)}|z|^{1+\lambda}\left(1+\frac{\beta|b|}{[n+1]_{q}\left[1+\alpha\left([n+1]_{q}-1\right)\right]}|z|^{n}\right) .
\end{aligned}
$$

The result is sharp for the function $f(z)$ given by (5.10).

Putting $\beta=1$ in Corollaries 14 and 15, respectively, we have the following corollaries.

Corollary 16. Let $f(z) \in \mathcal{G}_{n}^{\alpha}(\lambda, b, q)$. Then

$$
\begin{aligned}
& \frac{\Gamma_{q}(2)}{\Gamma_{q}(2-\lambda)}|z|^{1-\lambda}\left(1-\frac{|b|}{[n+1]_{q}\left[1+\alpha\left([n+1]_{q}-1\right)\right]}|z|^{n}\right) \\
& \quad \leqq\left|D_{q, z}^{\lambda} f(z)\right| \\
& \quad \leqq \frac{\Gamma_{q}(2)}{\Gamma_{q}(2-\lambda)}|z|^{1-\lambda}\left(1+\frac{|b|}{[n+1]_{q}\left[1+\alpha\left([n+1]_{q}-1\right)\right]}|z|^{n}\right) .
\end{aligned}
$$

The result is sharp for the function $f(z)$ given by (5.10) with $\beta=1$.

Corollary 17. Let $f(z) \in \mathcal{E}_{n}^{\alpha}(\lambda, \beta, b, q)$. Then

$$
\begin{aligned}
& \frac{\Gamma_{q}(2)}{\Gamma_{q}(2+\lambda)}|z|^{1+\lambda}\left(1-\frac{|b|}{[n+1]_{q}\left[1+\alpha\left([n+1]_{q}-1\right)\right]}|z|^{n}\right) \\
& \quad \leqq\left|I_{q, z}^{\lambda} f(z)\right| \\
& \quad \leqq \frac{\Gamma_{q}(2)}{\Gamma_{q}(2+\lambda)}|z|^{1+\lambda}\left(1+\frac{|b|}{[n+1]_{q}\left[1+\alpha\left([n+1]_{q}-1\right)\right]}|z|^{n}\right) .
\end{aligned}
$$

The result is sharp for the function $f(z)$ given by (5.10) with $\beta=1$.

Remark 3. The results asserted by Corollaries 15 and 16 provide, respectively, the corrected versions of the results obtained by Purohit and Raina [12, Corollaries 3 and 4].

Remark 4. Putting $\lambda=0$ in our results, we obtain a number of new results for the function classes $\gamma_{n}^{\alpha}(\beta, b, q)$ and $\mathcal{E}_{n}^{\alpha}(\beta, b, q)$. 


\section{CONCLUSion}

In our present investigation, we applied various operators of $q$-calculus and fractional $q$-calculus in the study of two general subclasses $\wp_{n}^{\alpha}(\lambda, \beta, b, q)$ and $\mathcal{E}_{n}^{\alpha}(\lambda, \beta, b, q)$ of normalized analytic functions with complex order and negative coefficients. For each of these function classes, we have derived their associated coefficient estimates, radii of close-to-convexity, starlikeness and convexity, extreme points, and growth and distortion theorems. Our main results and their new or known consequences are stated and proved as theorems and corollaries.

\section{REFERENCES}

[1] S. Abelomen, K. A. Selvakumaran, M. M. Rashidi, and S. D. Purohit, "Subordination conditions for a class of non-Bazilavić type defined by using fractional $q$-culculus operators." Facta Univ. Ser. Math. Inform., vol. 32, pp. 255-267, 2017, doi: 10.22190/FUMI1702255A.

[2] M. H. Abu-Risha, M. H. Annaby, M. E.-H. Ismail, and S. Mansour, "Linear $q$-difference equations." Z. Anal. Anwend., vol. 26, pp. 481-494, 2007, doi: 10.4171/ZAA/1338.

[3] O. Altintaş and Ö. Özkan, "Neighborhoods of a class of analytic functions with negative coefficients." Appl. Math. Lett., vol. 13, no. 3, pp. 63-67, 2000, doi: 10.1016/S0893-9659(99)00187-1.

[4] M. H. Annaby and Z. S. Mansour, q-Fractional Calculus and Equations. New York: Springer, 2012. doi: 10.1007/978-3-642-30898-7.

[5] M. K. Aouf, "Neighborhoods of a certain family of multivalent functions defined by using a fractional derivative operator." Bull. Belgian Math. Soc. Simon Stevin, vol. 16, pp. 31-40, 2009, doi: 10.1016/S0893-9659(99)00187-1.

[6] M. K. Aouf, H. E. Darwish, and G. S. Sălăgean, "On a generalization of starlike functions with negative coefficients." Mathematica (Cluj), vol. 43, no. 66, pp. 3-10, 2001, doi: 10.1016/S08939659(99)00187-1.

[7] M. K. Aouf and J. Dziok, "Distortion and convolutional theorems for operators of generalized fractional calculus involving Wright function." J. Appl. Anal., vol. 14, pp. 183-192, 2008, doi: 10.1515/JAA.2008.183.

[8] G. Gasper and M. Rahman, Basic Hypergeometric Series. Cambridge, London and New York: (with a Foreword by Richard Askey), Encyclopedia of Mathematics and Its Applications, Vol. 35, Second edition, Cambridge University Press, 2004.

[9] F. H. Jackson, "On $q$-functions and a certain difference operator." Trans. Roy. Soc. Edinburgh, vol. 46, pp. 64-72, 1908, doi: 10.1515/JAA.2008.183.

[10] S. Mahmood, N. Raza, E. S. A. Abujarad, G. Srivastava, H. M. Srivastava, and S. N. Malik, "Geometric properties of certain classes of analytic functions associated with a $q$-integral operator." Symmetry, vol. 11, no. Article ID 719, pp. 1-14, 2019, doi: 10.3390/sym11050719.

[11] S. Owa and H. M. Srivastava, "Univalent and starlike generalized hypergeometric functions." Canad. J. Math., vol. 39, pp. 1057-1077, 1987, doi: 10.4153/CJM-1987-054-3.

[12] S. D. Purohit and R. K. Raina, "Certain subclasses of analytic functions associated with fractional calculus operators." Math. Scand., vol. 109, pp. 55-70, 2011, doi: 10.7146/math.scand.a-15177.

[13] S. D. Purohit and R. K. Raina, "Fractional $q$-calculus and certain subclasses of univalent analytic functions." Mathematica (Cluj), vol. 55, no. 78, pp. 62-74, 2013.

[14] K. A. Selvakurmaran, S. D. Purohit, A. Secer, and M. Bayram, "Convexity of certain $q$-integral operators of p-valent functions." Abstr. Appl. Anal., vol. 2014, no. Article ID 925902, pp. 1-7, 2014, doi: 10.1155/2014/925902. 
[15] T. M. Seoudy and M. K. Aouf, "Convolution properties for certain classes of analytic functions defined by $q$-derivative operator.” Abstr. Appl. Anal., vol. 2014, no. Article ID 846719, pp. 1-7, 2014, doi: 10.1155/2014/846719.

[16] T. M. Seoudy and M. K. Aouf, "Coefficient estimates of new class of $q$-starlike and $q$-convex functions of complex order." J. Math. Inequal., vol. 10, pp. 135-145, 2016, doi: 10.7153/jmi-1011.

[17] L. Shi, Q. Khan, G. Srivastava, J.-L. Liu, and M. Arif, "A study of multivalent $q$-starlike functions connected with circular domain." Mathematics, vol. 7, no. Article ID 670, pp. 1-12, 2019, doi: $10.3390 /$ math7080670.

[18] H. M. Srivastava and M. K. Aouf, "A certain fractional derivative operator and its applications to a new class of analytic and multivalent functions with negative coefficients." J. Math. Anal. Appl., vol. 171, pp. 1-13, 1992, doi: 10.1016/0022-247X(92)90373-L.

[19] H. M. Srivastava and M. K. Aouf, “ A certain fractional derivative operator and its applications to a new class of analytic and multivalent functions with negative coefficients. II." J. Math. Anal. Appl., vol. 171, pp. 673-688, 1995, doi: 10.1006/jmaa.1995.1197.

[20] H. M. Srivastava and M. K. Aouf, "Some applications of fractional calculus operators to certain subclasses of prestarlike functions of negative coefficients." J. Math. Anal. Appl., vol. 30, pp. 53-61, 1995, doi: 10.1016/0898-1221(95)00067-9.

[21] H. M. Srivastava, B. Khan, N. Khan, and Q. Z. Ahmad, "Coefficient inequalities for $q$-starlike functions associated with the Janowski functions." Hokkaido Math. J., vol. 48, pp. 407-425, 2019, doi: $10.1155 / 2018 / 8492072$.

[22] H. M. Srivastava, A. O. Mostafa, M. K. Aouf, and H. M. Zayed, "Basic and fractional $q-$ calculus and associated Fekete-Szegö problem for $p$-valently $q$-starlike functions and $p$-valently $q$-convex functions of complex order.” Miskolc Math. Notes, vol. 20, pp. 489-509, 2019, doi: 10.18514/MMN.2019.2405.

[23] H. M. Srivastava and S. Owa (Editors), Univalent Functions, Fractional Calculus, and Their Applications. New York, Chichester, Bribane and Toronto: Halsted Press (Ellis Horwood Limited, Chichester), John Wiley and Sons, 1989.

[24] H. M. Srivastava and S. Owa (Editors), Current Topics in Analytic Function Theory. Singapore, New Jersey, London and Hong Kong: World Scientific Publishing Company, 1992.

[25] B. Wongsaijai and N. Sukantamala, "Applications of fractional $q$-calculus to certain subclass of analytic $p$-valent functions with negative coefficients." Abstr. Appl. Anal., vol. 2015, no. Article ID 273236, pp. 1-12, 2015, doi: 10.1155/2015/273236.

\section{Authors' addresses}

\section{H. M. Srivastava}

Department of Mathematics and Statistics, University of Victoria, Victoria, British Columbia V8W 3R4, Canada and Department of Medical Research, China Medical University Hospital, China Medical University, Taichung 40402, Taiwan, Republic of China and Department of Mathematics and Informatics, Azerbaijan University, 71 Jeyhun Hajibeyli Street, AZ1007 Baku, Azerbaijan

E-mail address: harimsri@math.uvic.ca

\section{K. Aouf}

Department of Mathematics, Faculty of Science, Mansoura University, Mansoura 35516, Egypt

E-mail address: mkaouf127@yahoo.com

\section{A. O. Mostafa}

Department of Mathematics, Faculty of Science, Mansoura University, Mansoura 35516, Egypt

E-mail address: adelaeg254@yahoo.com 